\title{
Evidence of RNAi in humans from systemically administered siRNA via targeted nanoparticles
}

\author{
Mark E. Davis ${ }^{1}$, Jonathan E. Zuckerman ${ }^{1}$, Chung Hang J. Choi ${ }^{1}$, David Seligson ${ }^{2,3}$, Anthony Tolcher ${ }^{5}$, \\ Christopher A. Alabi ${ }^{1} \dagger$, Yun Yen ${ }^{6}$, Jeremy D. Heidel ${ }^{7} \&$ Antoni Ribas ${ }^{2,4}$
}

\begin{abstract}
Therapeutics that are designed to engage RNA interference (RNAi) pathways have the potential to provide new, major ways of imparting therapy to patients ${ }^{1,2}$. Long, double-stranded RNAs were first shown to mediate RNAi in Caenorhabditis elegans ${ }^{3}$, and the potential use of RNAi for human therapy has been demonstrated by the finding that small interfering RNAs (siRNAs; approximately 21base-pair double-stranded RNA) can elicit RNAi in mammalian cells without producing an interferon response ${ }^{4}$. We are at present conducting the first in-human phase I clinical trial involving the systemic administration of siRNA to patients with solid cancers using a targeted, nanoparticle delivery system. Here we provide evidence of inducing an RNAi mechanism of action in a human from the delivered siRNA. Tumour biopsies from melanoma patients obtained after treatment show the presence of intracellularly localized nanoparticles in amounts that correlate with dose levels of the nanoparticles administered (this is, to our knowledge, a first for systemically delivered nanoparticles of any kind). Furthermore, a reduction was found in both the specific messenger RNA (M2 subunit of ribonucleotide reductase (RRM2)) and the protein (RRM2) levels when compared to pre-dosing tissue. Most notably, we detect the presence of an mRNA fragment that demonstrates that siRNA-mediated mRNA cleavage occurs specifically at the site predicted for an RNAi mechanism from a patient who received the highest dose of the nanoparticles. Together, these data demonstrate that siRNA administered systemically to a human can produce a specific gene inhibition (reduction in mRNA and protein) by an RNAi mechanism of action.
\end{abstract}

A major challenge with the use of siRNAs in mammals is their intracellular delivery to specific tissues and organs that express the target gene. The first demonstrations of siRNA-mediated gene silencing in mammals through systemic administration were accomplished using naked siRNA and methods of administration not compatible with clinical application ${ }^{5-7}$. Since then, several delivery vehicles have been combined with siRNAs to improve their delivery in animal models ${ }^{1,2}$. A recent study provided direct evidence for the siRNA mechanism of action by using a modified 5'-RACE (rapid amplification of complementary DNA ends) PCR technique providing positive identification of the specific mRNA cleavage product ${ }^{8}$. Human clinical trials with synthetic siRNAs began in 2004, using direct intraocular siRNA injections for patients with blinding choroidal neovascularization (CNV). Subsequently, other clinical trials have initiated ${ }^{2}$ and early clinical data are beginning to appear ${ }^{9,10}$. Although there are animal studies that do support the possibility of an RNAi mechanism of action from administered siRNA ${ }^{11}$, other animal data from siRNAs injected into the eyes of mice for the treatment of CNV suggest non-RNAi mechanisms of action for CNV suppression ${ }^{12}$. At this time, no direct evidence for an RNAi mechanism of action in humans from siRNA administered either locally or systemically has been reported.

We are at present conducting the first, to our knowledge, siRNA clinical trial that uses a targeted nanoparticle-delivery system (clinical trial registration number NCT00689065) ${ }^{13}$. Patients with solid cancers refractory to standard-of-care therapies are administered doses of targeted nanoparticles on days 1, 3, 8 and 10 of a 21-day cycle by a 30 -min intravenous infusion. The nanoparticles consist of a synthetic delivery system (Fig. 1a) containing: (1) a linear, cyclodextrin-based polymer (CDP), (2) a human transferrin protein (TF) targeting ligand displayed on the exterior of the nanoparticle to engage TF receptors (TFR) on the surface of the cancer cells, (3) a hydrophilic polymer (polyethylene glycol (PEG) used to promote nanoparticle stability in biological fluids), and (4) siRNA designed to reduce the expression of the RRM2 (sequence used in the clinic was previously denoted siR2B +5$)^{14}$. The TFR has long been known to be upregulated in malignant cells ${ }^{15}$, and RRM2 is an established anti-cancer target ${ }^{16}$. These nanoparticles (clinical version denoted as CALAA-01) have been shown to be well tolerated in multi-dosing studies in non-human primates ${ }^{17}$. Although a single patient with chronic myeloid leukaemia has been administered siRNA by liposomal delivery ${ }^{18}$, our clinical trial is the initial human trial to systemically deliver siRNA with a targeted delivery system and to treat patients with solid cancer ${ }^{13}$.

To ascertain whether the targeted delivery system can provide effective delivery of functional siRNA to human tumours, we investigated biopsies from three patients from three different dosing cohorts; patients A, B and C, all of whom had metastatic melanoma and received CALAA- 01 doses of 18,24 and $30 \mathrm{mg} \mathrm{m}^{-2}$ siRNA, respectively. Given the highly experimental nature of this protocol, the regulatory process at both the local and federal levels explicitly precluded a provision for mandatory biopsies in all patients. Therefore, biopsies were obtained on a voluntary basis. Biopsies in these three patients were collected after the final dose of cycle 1 (denoted $\mathrm{A}_{\text {post }}, \mathrm{B}_{\text {post }}$ and $\mathrm{Cl}_{\text {post }}$ ) and compared to archived tissue (denoted $\mathrm{A}_{\text {pre }}, \mathrm{B}_{\text {pre }}$ and $\mathrm{C}_{\text {pre }}$ ). Patient $\mathrm{C}$ continued therapy beyond one cycle and provided another set of biopsy material $(\mathrm{C} 2$ pre that was obtained approximately 1 month after the final dose of cycle 1 , and $\mathrm{C} 2$ post that was collected on the day of the final dose of cycle 2). Because of the limited sample amount, only immunohistochemistry (IHC) and staining for the nanoparticles could be performed on the $\mathrm{C} 1_{\text {pre }}$ and $\mathrm{C} 1_{\text {post }}$ samples, and protein (for western blot analyses) was only available from the $\mathrm{C} 2$ pre and $\mathrm{C} 2$ post samples. Details of this clinical trial will be reported elsewhere when completed. 
a

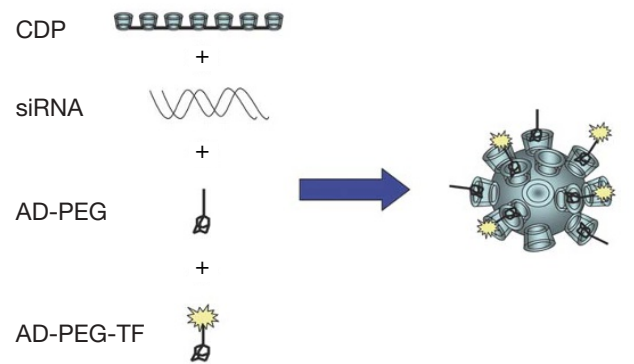

b
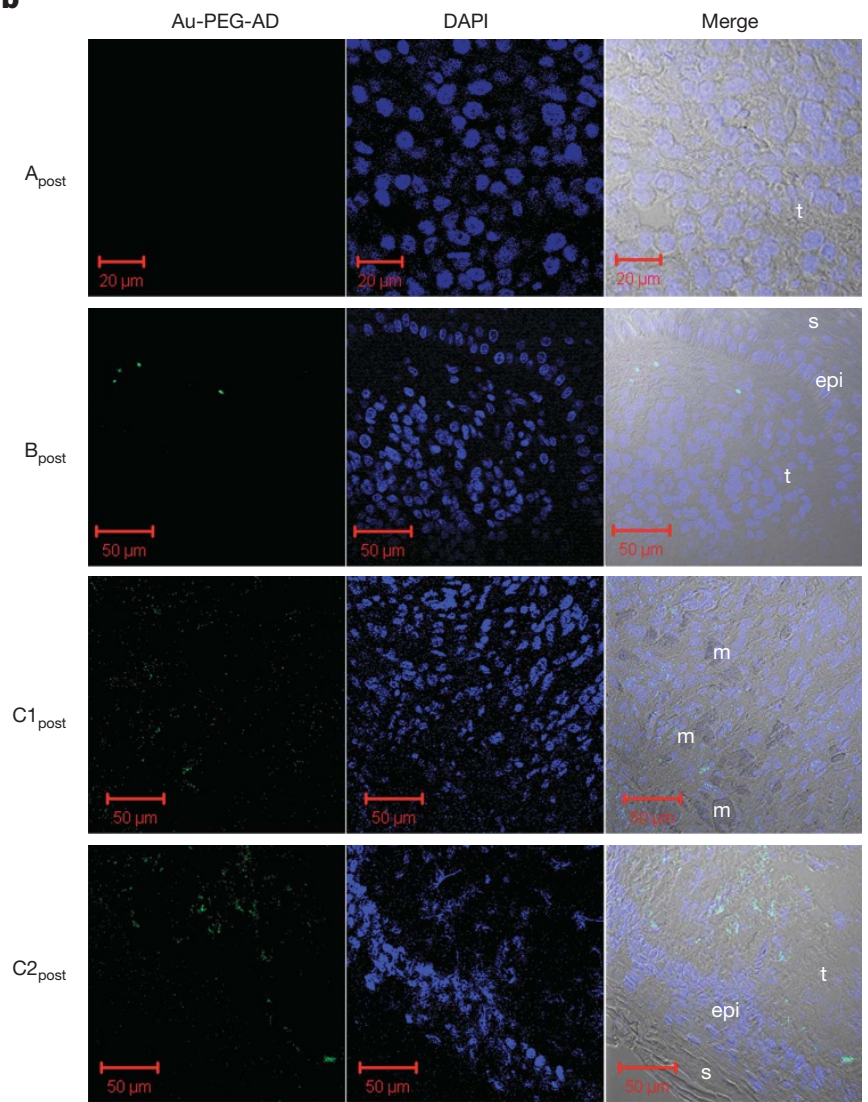

Figure 1 | Detection of targeted nanoparticles in human tumours.

a, Schematic representation of the targeted nanoparticles. The polyethylene glycol (PEG) molecules are terminated with adamantane (AD) that form inclusion complexes with surface cyclodextrins to decorate the surface of the nanoparticle with PEG for steric stabilization and PEG-TF for targeting.

b, Confocal images of post-treatment biopsy sections from patients A, B and C. Left, Au-PEG-AD stain; middle, DAPI stain; right, merged images of the left and right panels with the bright field. epi, epidermis, m, melanophage; $\mathrm{s}$, skin side; $\mathrm{t}$, tumour side.

The targeted nanoparticles (about 70-nm diameter) were administered intravenously, as they are designed to circulate and then to accumulate and permeate in solid tumours ${ }^{13}$. Within the tumour, the TF-targeting ligand assists in directing the nanoparticles into tumour cells overexpressing TFR ${ }^{19}$. To detect the nanoparticles in tumour cells, sections of the tumour tissue were stained for the presence of the nanoparticles using a 5-nm gold particle that is capped with thiolated PEG containing adamantane $(\mathrm{AD})$ at the end distal to the thiol (AD-PEG-Au) to allow for multivalent binding to the cyclodextrins (Supplementary Scheme 2). The function of the stain has been previously confirmed using other cyclodextrin-containing particles ${ }^{20}$, and is demonstrated here for the targeted nanoparticles carrying siRNA in vitro (Supplementary Fig. 1) and in vivo (Supplementary Figs 2 and 3). Transmission electron microscopy (TEM) images of the nanoparticles confirm that in mice, the nanoparticles are intracellular (Supplementary Fig. 2). Samples A, B and C1, analysed in a blinded fashion, demonstrated a heterogeneous distribution of nanoparticles only in post-dosing tumour tissue (Fig. 1 for post-dosing and Supplementary Fig. 4 for pre-dosing). The nanoparticles can localize intracellularly in tumour tissue and are not found in the adjacent epidermis (Fig. 1). In these biopsies TEM images were dominated by melanosomes ${ }^{21}$ inhibiting the identification of the nanoparticles (data not shown). Samples $\mathrm{C}_{\text {post }}$ and $\mathrm{C} 2_{\text {post }}$ show the highest number and intensity of stained regions, $B_{\text {post }}$ shows a decreased amount of staining relative to samples $\mathrm{Cl}_{\text {post }}$ and $\mathrm{C} 2_{\text {post }}$ (Fig. 1b), $\mathrm{A}_{\text {post }}$ does not show the presence of the stain (Fig. 1b), and all the pre-dosing samples are completely negative for the stain (Supplementary Fig. 4). This is the first example of a dosedependent accumulation of targeted nanoparticles in tumours of humans from systemic injections for nanoparticles of any type.

Tumour RRM2 mRNA levels were measured by quantitative realtime reverse-transcriptase polymerase chain reaction (qRT-PCR) and were performed in a blinded fashion ${ }^{22}$. Reduction in RRM2 mRNA is observed in the post-treatment samples (Fig. 2). Because samples $A_{\text {pre }}$ and $B_{\text {pre }}$ are from tissues collected many months before the initiation of siRNA treatment, the fraction of the overall reduction in mRNA observed in $A_{\text {post }}$ and $B_{\text {post }}$ attributable to the nanoparticle treatment cannot be directly ascertained. Unfortunately, we were not able to perform PCR on the $\mathrm{C} 1$ samples. However, the PCR data from the $\mathrm{C} 2$ pre and $\mathrm{C} 2$ post samples (collected 10 days apart) provide direct evidence for RRM 2 mRNA reduction by the treatment of the patient with the nanoparticles.

To ascertain whether the RRM2 protein level is reduced in the tumour because of the siRNA treatment, IHC and western blotting were used as previously described in mice ${ }^{23}$. Because RRM2 protein expression is largely restricted to the late G1/early S phase of the cell cycle, not all of the tumour cells will be expressing RRM2. Figure 3 shows IHC data for RRM2 and TFR proteins in $C 1_{\text {pre }}$ and $C 1_{\text {post }}$ samples (IHC analyses were performed in a blinded fashion and ten random regions of each sample were analysed). Considerable reduction in RRM2 is observed (mean scoring of RRM2 from the ten sections was reduced fivefold) after treatment, whereas TFR levels are slightly increased (mean scoring of TFR from the ten sections was increased 1.2-fold) in the $\mathrm{Cl}_{\text {pre }}$ and $\mathrm{Cl}_{\text {post }}$ samples. The low level of RRM2 that is observed by IHC in the $C 1_{\text {post }}$ sample is maintained in the $\mathrm{C} 2$ pre and $\mathrm{C} 2$ post samples (by IHC). Western blot analyses of the $\mathrm{C} 2$ pre and $\mathrm{C} 2$ post samples show a reduction in the level of the RRM2 protein that is due to the siRNA treatment ( $R R M 2$ mRNA reductions exceeded the reduction levels obtained from protein but this could be due to post-transcriptional mechanisms that have been observed
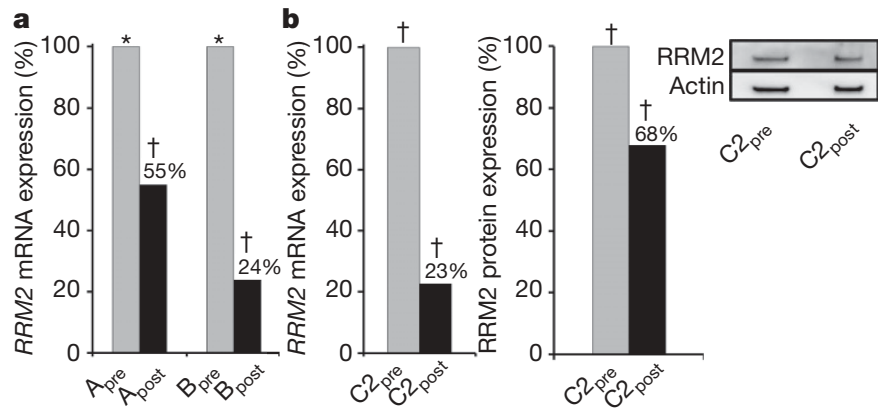

Figure 2 | RRM2 mRNA and protein expression in tumour tissue. a, qRT-PCR analysis of RRM2 mRNA levels in samples from patients $\mathrm{A}$ and $\mathrm{B}$ before and after dosing. RRM2 mRNA levels are normalized to TATA box binding protein $(T B P)$ mRNA levels. Results are presented as the percentage of the pre-dosing $R R M 2 / T B P$ mRNA levels for each patient. b, qRT-PCR and western blot analysis of RRM2 protein expression from patient samples $\mathrm{C} 2$ pre and $C 2$ post. The bar graph shows the average volume of western blot bands from two independent experiments; one representative blot is pictured. Asterisk denotes archived samples; dagger symbol denotes samples obtained during the trial. 


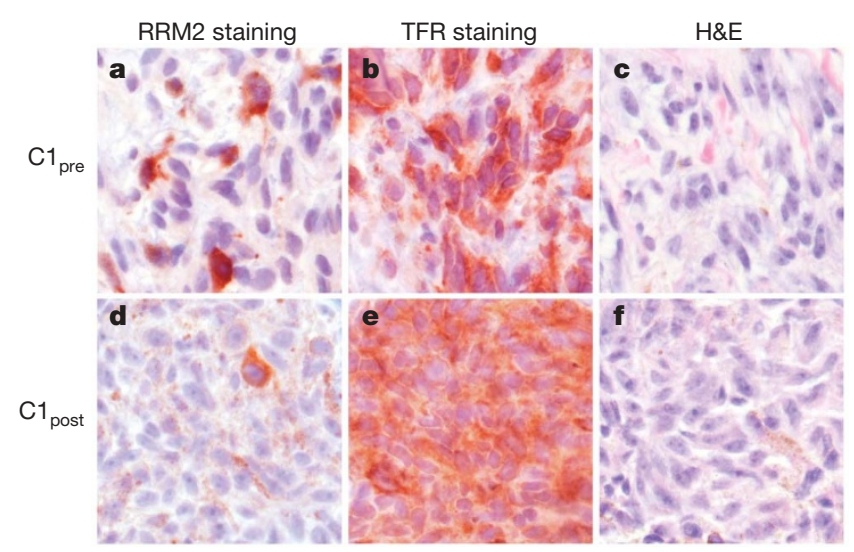

Figure 3 | RRM2 and TFR protein expression in $\mathbf{C 1}_{\text {pre }}$ and $\mathbf{C 1}_{\text {post }}$ samples. a-f, Photomicrographs of malignant melanoma belonging to pre-treatment $(\mathbf{a}-\mathbf{c})$ and post-treatment $(\mathbf{d}-\mathbf{f})$ samples. Protein expression is represented as brick-red (Nova Red) chromagen staining in immunohistochemically treated slides (RRM2 (a, d) and TFR $(\mathbf{b}, \mathbf{e}))$. The same tissues are also stained with haematoxylin and eosin (H\&E; c, f). Brown, diffuse, finely granular colour seen in the post-treatment images $(\mathbf{d}-\mathbf{f})$ is the endogenous pigment of this lightly melanized tumour. Original magnification used, $\times 40$.

previousl $\left.y^{24}\right)$. The decreases in the RRM2 mRNA and protein observed after treatment (Fig. 2b) suggest the siRNA treatment remains effective after several cycles of dosing. The IHC data from patient A do not show changes in RRM2 expression after dosing, whereas results from patient $B$ are indicative of reductions in maximal RRM2 expression (IHC scoring of the regions of maximal expression showed a 1.5-fold decrease), but the overall mean expression levels remained relatively constant (IHC scoring of the ten sections).

To demonstrate that the siRNA delivered by the targeted nanoparticles can engage the RNAi machinery, the mRNA cleavage products were characterized using a modified 5'-RNA-ligand-mediated
RACE (5'-RLM-RACE) PCR technique (Fig. 4). An RRM2 mRNA fragment-the $5^{\prime}$ end of which matches the predicted cleavage site (ten base pairs (bp) from the $5^{\prime}$ end of the antisense strand) - was detected in the $\mathrm{C} 2_{\text {pre }}$ and $\mathrm{C} 2_{\text {post }}$ samples, but not in $\mathrm{B}_{\text {post }}$ and $\mathrm{A}_{\text {post }}$ or their corresponding pre-treatment samples. RACE does not provide a quantitative measure of the amount of the fragments so the intensities of the bands cannot be correlated with the amounts in the tissue samples. The presence of this $R R M 2 \mathrm{mRNA}$ fragment from patient $\mathrm{C}$ indicates that siRNA delivered by targeted nanoparticles can engage the RNAi machinery in a solid human tumour and induce the desired mRNA cleavage. Furthermore, this result indicates that at least a portion of the RRM2 mRNA and protein reductions observed from the $\mathrm{C} 2$ samples are due to a bona fide RNAi mechanism. The presence of the RRM2 mRNA fragment in the $\mathrm{C} 2$ pre sample suggests that siRNA can provide an RNAi mechanism for several weeks (mRNA cleavage in the $\mathrm{C}_{2}$ pre sample must originate from cycle one dosing) as the RRM2 protein levels remained relatively constant when compared to the $\mathrm{Cl}_{\text {post }}$ sample (IHC). We have shown that the length of the RNAi effects of delivered siRNA in both cells and animals (mice) is dependent on the doubling time of the cells being analysed (longer inhibition times with longer cell doubling times) ${ }^{25}$. Gene silencing by siRNA can occur on the timescale observed here, approximately 1 month, provided the cell doubling times are long ${ }^{25}$. Patient $\mathrm{C}$ had stable disease between these biopsies, and these mostly quiescent tumours have very slow growth kinetics that would be suitable to experience lengthy RNAi effects ${ }^{25}$. Furthermore, we do not know how long the nanoparticles reside within the cells and release siRNA. Because the nanoparticles are observed in the sample $\mathrm{Cl}_{\text {post }}$ and not the sample $\mathrm{C} 2$ pre, they must disassemble within 1 month. Thus, the pharmacodynamics of the RNAi effects could be due to the combination of the nanoparticle disassembly time and the time that the siRNA resides within the RNAi machinery.

When taken together, the data presented here provide the first, to our knowledge, mechanistic evidence of RNAi in a human from an

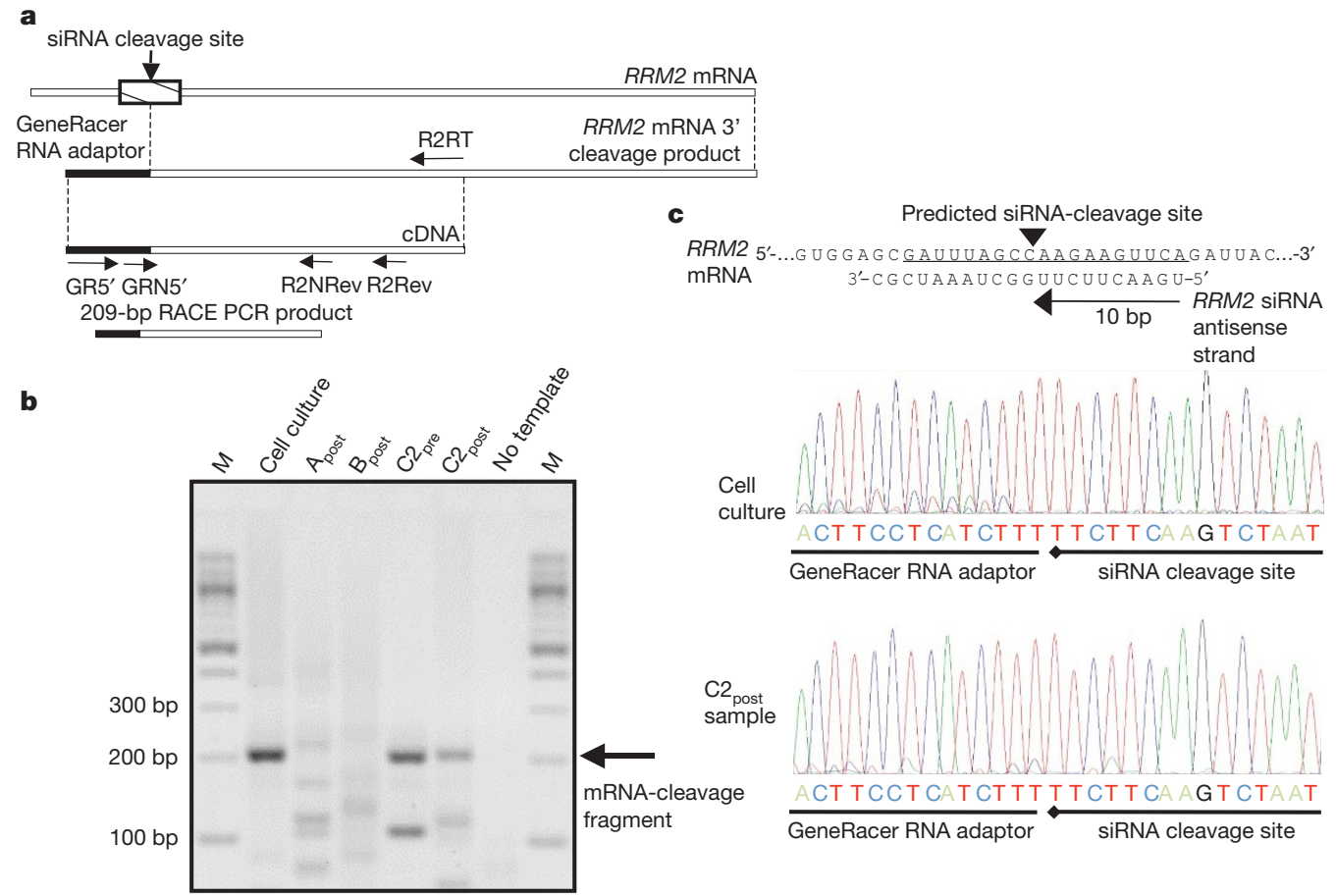

Figure 4 | 5'-RLM-RACE detection of siRNA-induced mRNA cleavage fragment. a, Schematic depicting the location of the predicted anti-RRM2 siRNA cleavage site and the primers used for PCR amplification of the cleavage fragment. GR5', GeneRacer 5' primer; GRN5', GeneRacer 5' nested primer; R2RT, RRM2 gene-specific reverse-transcriptase primer; R2Rev, $R R M 2$ gene-specific reverse primer; R2NRev, $R R M 2$ gene-specific nested primer. b, Agarose gel of 5'-RLM-RACE PCR amplification products from patient samples $\left(\mathrm{A}_{\text {post }}, \mathrm{B}_{\text {post }}, \mathrm{C} 2_{\text {pre }}\right.$ and $\left.\mathrm{C} 2_{\text {post }}\right)$ and the in vitro positive control (cell culture). M, molecular mass ladder. c, The RRM2 mRNA sequence and siRNA antisense strand are illustrated to show where the cleavage occurs with an RNAi mechanism. The sequence chromatographs obtained from an in vitro cell culture experiment with HT-144 melanoma cells and the patient $\mathrm{C} 2_{\text {post }}$ sample are illustrated. 
administered siRNA. Moreover, these data demonstrate the first example of dose-dependent accumulation of targeted nanoparticles in human tumours. The reduction of the RRM 2 mRNA and protein by the RRM2-specific siRNA is observed, and the results from 5 '-RLM-RACE analyses show that the delivered siRNA engages the RNAi machinery. These data demonstrate that RNAi can occur in a human from a systemically delivered siRNA, and that siRNA can be used as a gene-specific therapeutic.

\section{METHODS SUMMARY}

Detection of targeted nanoparticles in biopsy samples. Snap-frozen patient biopsy samples were embedded in Tissue-Tek O.C.T. compound (Sakura) for the generation of $6-\mu \mathrm{m}$-thick cryosections. After immersion in PBS at $37^{\circ} \mathrm{C}$ for $1 \mathrm{~h}$ to remove any surface O.C.T., and subsequent fixation with acetone at $-20^{\circ} \mathrm{C}$ for $20 \mathrm{~min}$ to permeabilize the cell membrane, sections received staining of PEGylated, AD-modified gold nanoparticles (Au-PEG-AD; see Supplementary Information for a description of their preparation) in the dark for $2 \mathrm{~h}$. Brief rinses with PBS were used to remove any non-specifically bound Au-PEG-AD before mounting with ProLong Gold antifade reagent and staining with 4',6-diamidino-2-phenylindole (DAPI; Invitrogen). A Zeiss LSM 510 confocal scanning microscope was used to collect the images (DAPI-excitation: $370 \mathrm{~nm}$, emission: $440 \mathrm{~nm}$; Au-PEG-AD-excitation: $488 \mathrm{~nm}$, emission: $507 \mathrm{~nm}$ ).

5'-RLM-RACE. 5'-RLM-RACE was performed according to the Invitrogen GeneRacer manual with modifications. Two-to-eight micrograms of total RNA was ligated directly to $250 \mathrm{ng}$ GeneRacer RNA adaptor using T4 RNA ligase. After phenol extraction and ethanol precipitation, the purified ligation products were reverse-transcribed using SuperScriptIII (Invitrogen) and an RRM2 genespecific reverse-transcription primer (5'-CTCTCTCCTCCGATGGTTTG-3'). $5^{\prime}$-RLM-RACE PCR was performed using the GeneRacer $5^{\prime}$ and an RRM2 gene-specific reverse primer ( $5^{\prime}$-GGCCAGGCATCAGTCCTCGTTTCTTG-3'). PCR was performed using a Bio-Rad MJ Mini personal thermocycler using PCR conditions of $95^{\circ} \mathrm{C}$ for $3 \mathrm{~min}(1 \mathrm{cycle}), 95^{\circ} \mathrm{C}$ for $30 \mathrm{~s}, 60^{\circ} \mathrm{C}$ for $30 \mathrm{~s}, 72^{\circ} \mathrm{C}$ for $1 \mathrm{~min}$ ( 40 cycles), $72^{\circ} \mathrm{C}$ for $10 \mathrm{~min}$ ( 1 cycle). A second round of nested PCR was then performed using the GeneRacer 5 ' nested primer and an RRM2 gene-specific nested primer ( $5^{\prime}$-GGCCCAGTCTGCCTTCTTCTTGAC- $\left.3^{\prime}\right)$. PCR products were run on a $2 \%$ agarose gel and stained with $1 \mu \mathrm{g} \mathrm{\mu l^{-1 }}$ ethidium bromide. PCR products were excised from the gel and sequenced directly to confirm RACE band identities.

Full Methods and any associated references are available in the online version of the paper at www.nature.com/nature.

\section{Received 23 September 2009; accepted 1 March 2010.}

\section{Published online 21 March 2010.}

1. Bumcrot, D., Manoharan, M., Koteliansky, V. \& Sah, D. W. Y. RNAi therapeutics: a potential new class of pharmaceutical drugs. Nature Chem. Biol. 2, 711-719 (2006).

2. Castanotto, D. \& Rossi, J. J. The promises and pitfalls of RNA-interference-based therapeutics. Nature 457, 426-433 (2009).

3. Fire, A. et al. Potent and specific genetic interference by double-stranded RNA in Caenorhabditis elegans. Nature 391, 806-811 (1998).

4. Elbashir, S. M. et al. Duplexes of 21-nucleotide RNAs mediate RNA interference in cultured mammalian cells. Nature 411, 494-498 (2001).

5. McCaffrey, A. P. et al. RNA interference in adult mice. Nature 418, 38-39 (2002).

6. Lewis, D. L., Hagstrom, J. E., Loomis, A. G., Wolff, J. A. \& Herweijer, H. Efficient delivery of siRNA for inhibition of gene expression in postnatal mice. Nature Genet. 32, 107-108 (2002).

7. Song, E. et al. RNA interference targeting Fas protects mice from fulminant hepatitis. Nature Med. 9, 347-351 (2003).

8. Soutschek, J. et al. Therapeutic silencing of an endogenous gene by systemic administration of modified siRNAs. Nature 432, 173-178 (2004).

9. DeVincenzo, J. et al. Evaluation of the safety, tolerability and pharmacokinetics of ALN-RSV01, a novel RNAi antiviral therapeutic directed against respiratory syncytial virus (RSV). Antiviral Res. 77, 225-231 (2008).

10. Leachman, S. A. et al. First-in-human mutation-targeted siRNA phase lb trial of an inherited skin disorder. Mol. Ther. 18, 442-446 (2010).
11. Alvarez, R. et al. RNA interference-mediated silencing of the respiratory syncytial virus nucleocapsid defines a potent anti-viral strategy. Antimicrob. Agents Chemother. 53, 3952-3962 (2009).

12. Kleinman, M. E. et al. Sequence- and target-independent angiogenesis suppression by siRNA via TLR3. Nature 452, 591-597 (2008).

13. Davis, M. E. The first targeted delivery of siRNA in humans via a self-assembling, cyclodextrin polymer-based nanoparticle: from concept to clinic. Mol. Pharm. 6, 659-668 (2009)

14. Heidel, J. D. et al. Potent siRNA inhibitors of ribonucleotide reductase subunit RRM2 reduce cell proliferation in vitro and in vivo. Clin. Cancer Res. 13, 2207-2215 (2007).

15. Gatter, K. C., Brown, G., Trowbridge, I. S., Wollston, R. E. \& Mason, D. Y. Transferrin receptors in human tissues: their distribution and possible clinical relevance. J. Clin. Pathol. 36, 539-545 (1983).

16. Cerqueira, N. M. F. S. A., Pereira, S., Fernandes, P. A. \& Ramos, M. J. Overview of ribonucleotide reductase inhibitors: an appealing target in anti-tumor therapy. Curr. Med. Chem. 12, 1283-1294 (2005).

17. Heidel, J. D. et al. Administration in non-human primates of escalating intravenous doses of targeted nanoparticles containing ribonucleotide reductase subunit M2 siRNA. Proc. Natl Acad. Sci. USA 104, 5715-5721 (2007).

18. Koldehoff, M., Steckel, N. K., Beelen, D. W. \& Elmaagacli, A. H. Therapeutic application of small interfering RNA directed against $b c r-a b$ t transcripts to a patient with imatinib-resistant chronic myeloid leukaemia. Clin. Exp. Med. 7, 47-55 (2007).

19. Bartlett, D. W., Su, H., Hildebrandt, I. J., Weber, W. A. \& Davis, M. E. Impact of tumor-specific targeting on the biodistribution and efficacy of siRNA nanoparticles measured by multimodality in vivo imaging. Proc. Natl Acad. Sci. USA 104, 15549-15554 (2007).

20. Schluep, T. et al. Pharmacokinetics and tumor dynamics of the nanoparticle IT-101 from PET imaging and tumor histological measurements. Proc. Natl Acad. Sci. USA 106, 11394-11399 (2009).

21. Riehl, R., Schartl, M. \& Kollinger, G. Comparative studies on the ultrastructure of malignant melanoma in fish and human by freeze-etching and transmission electron microscropy. J. Cancer Res. Clin. Oncol. 107, 21-31 (1984).

22. Juhasz, A., Vassilakos, A., Chew, H. K., Gandara, D. \& Yen, Y. Analysis of ribonucleotide reductase M2 mRNA levels in patient samples after GTI-2040 antisense drug treatment. Oncol. Rep. 15, 1299-1304 (2006).

23. Bartlett, D. W. \& Davis, M. E. Impact of tumor-specific targeting and dosing schedule on tumor growth inhibition after intravenous administration of siRNAcontaining nanoparticles. Biotechnol. Bioeng. 99, 975-985 (2008).

24. McClarty, G. A., Chan, A. K., Engstrom, Y., Wright, J. A. \& Thelander, L. Elevated expression of $\mathrm{M} 1$ and $\mathrm{M} 2$ components and drug-induced posttranscriptional modulation of ribonucleotide reductase in a hydroxyurea-resistant mouse cell line. Biochemistry 26, 8004-8011 (1987).

25. Bartlett, D. W. \& Davis, M. E. Insights into the kinetics of siRNA-mediated gene silencing from live-cell and live-animal bioluminescent imaging. Nucleic Acids Res. 34, 322-333 (2006).

Supplementary Information is linked to the online version of the paper at www.nature.com/nature.

Acknowledgements We thank H. Sazegar, E. Seja, A. Villanueva and the G-CRC nursing staff at University of California, Los Angeles (UCLA), and L. Kalinoski, J. Peterkin, S. Rele, Y. Liang and J. Y. C. Liu for their assistance in conducting the clinical trial. We thank J. S. Economou for performing the tumour biopsies, and B. Chmielowski and Z. Wainberg for patient referrals. Histotechnology support at the UCLA Biomarker Innovations Laboratory was provided by C. Savina and J. Reiss. We also thank J. Rossi for discussions on RACE analyses. This work was supported in part by the National Cancer Institute Grant CA U54 119347 and the Daljit S. \& Elaine Sarkaria Biomarker Laboratory Fund.

Author Contributions M.E.D., J.E.Z., A.R. and J.D.H. planned the experiments, J.E.Z., D.S., C.H.J.C., C.A.A., Y.Y., A.T. and A.R. conducted the experiments, M.E.D., J.E.Z., J.D.H., D.S., C.H.J.C. and A.R. analysed the data, and M.E.D., J.E.Z. and A.R. wrote the paper.

Author Information Reprints and permissions information is available at www.nature.com/reprints. The authors declare competing financial interests: details accompany the full-text HTML version of the paper at www.nature.com/ nature. Correspondence and requests for materials should be addressed to M.E.D. (mdavis@cheme.caltech.edu). 


\section{METHODS}

Detection of targeted nanoparticles in biopsy samples. Snap-frozen patient biopsy samples were embedded in Tissue-Tek O.C.T. compound (Sakura) to generate 6- $\mu \mathrm{m}$-thick cryosections. After immersion in PBS at $37^{\circ} \mathrm{C}$ for $1 \mathrm{~h}$ to remove any surface O.C.T., and in acetone at $-20^{\circ} \mathrm{C}$ for 20 min to permeabilize the cell membrane, sections received staining of PEGylated, adamantane-modified gold nanoparticles (Au-PEG-AD; see Supplementary Information for their preparation) in the dark for $2 \mathrm{~h}$. Brief rinses with PBS were used to remove any non-specifically bound Au-PEG-AD before mounting with ProLong Gold antifade reagent and staining with DAPI (Invitrogen). A Zeiss LSM 510 confocal scanning microscope (with a Plan-Neofluar $\times 40 / 0.75$ objective and up to $2 \times$ digital zoom) was used to collect the images (DAPI-excitation: $740 \mathrm{~nm}$ (two-photon laser), emission filter: 390-490 nm; Au-PEG-AD-excitation: 488 nm (argon laser), emission filter: bandpass (BP) $500-550 \mathrm{~nm}$ infrared radiation (IR)). The measured resolution at which images were acquired is $512 \times 512$ pixels, and the image bit-depth is 8 -bit. The Zeiss LSM Image Browser Software allows the extraction of images.

RNA extraction. Patient samples preserved in RNALater (Ambion) were suspended in TRIzol reagent (Invitrogen) and homogenized in a FastPrep-24 Tissue Homogenizer (MP Biomedicals). Total RNA was purified from the aqueous phase of TRIzol extract using the PureLink RNA Mini Kit (Invitrogen) following manufacturer recommendations. RNA was extracted from archived patient samples using RecoverAll total nucleic acid isolation kit (Ambion) following manufacturer instructions.

5'-RLM-RACE. 5'-RLM-RACE was performed according to the Invitrogen GeneRacer manual with modifications. Two-to-eight micrograms of total RNA was ligated directly to 250 ng GeneRacer RNA adaptor (5'-CGACUGGA GCACGAGGACACUGACAUGGACUGAAGGAGUAGAAA-3') using T4 RNA ligase (5 units) for $1 \mathrm{~h}$ at $37^{\circ} \mathrm{C}$. After phenol extraction and ethanol precipitation, the purified ligation products were reverse-transcribed using SuperScriptIII (Invitrogen) and an $R R M 2$ gene-specific reverse-transcription primer $\left(5^{\prime}\right.$-CT CTCTCCTCCGATGGTTTG- $3^{\prime}$ ) at $55^{\circ} \mathrm{C}$ for $45 \mathrm{~min}$ followed by inactivation at $70^{\circ}$ C. $5^{\prime}$-RLM-RACE-PCR was performed using the GeneRacer $5^{\prime}$ primer $\left(5^{\prime}\right.$-CGACTGGAGCACGAGGACACTGA- $\left.3^{\prime}\right)$ and an RRM2 gene-specific reverse primer ( $5^{\prime}$-GGCCAGGCATCAGTCCTCGTTTCTTG-3'). PCR was performed using a Bio-Rad MJ Mini personal thermocycler using PCR conditions of $95^{\circ} \mathrm{C}$ for $3 \mathrm{~min}$ ( 1 cycle), $95^{\circ} \mathrm{C}$ for $30 \mathrm{~s}, 60^{\circ} \mathrm{C}$ for $30 \mathrm{~s}, 72^{\circ} \mathrm{C}$ for $1 \mathrm{~min}$ ( 40 cycles), $72^{\circ} \mathrm{C}$ for $10 \mathrm{~min}(1 \mathrm{cycle})$. A second round of nested PCR was then performed using the GeneRacer $5^{\prime}$ nested primer ( $5^{\prime}$-GGACACTGACATGGACTGAAGG AGTA- $\left.3^{\prime}\right)$ and an RRM2 gene-specific nested primer (5'-GGCCCAGTCTGCCT TCTTCTTGAC- $3^{\prime}$ ). PCR products were run on a $2 \%$ agarose gel and stained with $1 \mu \mathrm{g} \mu \mathrm{l}^{-1}$ ethidium bromide. PCR products were excised from the gel and sequenced directly to confirm RACE band identities. For the cell culture RACE experiments, 500,000 HT-144 melanoma cells were transfected with $20 \mathrm{nM}$ RRM2 siRNA using Lipofectamine RNAiMax (Invitrogen). RNA was extracted for the RLM-RACE as described earlier, $48 \mathrm{~h}$ after transfection.

qRT-PCR. Patient RNA samples were reversed-transcribed using SuperScriptIII reverse transcriptase. White blood cell (WBC) cDNA (0.4-200 ng) was used as a
PCR template for standard curves of RRM2 and TBP. Two microlitres of prepared sample or standard cDNA was used for triplicate Taqman real-time PCR as described elsewhere ${ }^{25}$. Concentrations of RRM2 and TBP in the samples were calculated from the WBC cDNA standard curve, and RRM2 levels were normalized to TBP levels within the same sample.

Western blots. Total protein was recovered from the phenol/chloroform phase of TRIzol extraction (see description of the RNA extraction earlier). Samples were diluted to equivalent protein concentration and denatured by the addition of $\beta$-mercaptoethanol-containing Laemmli sample buffer. The primary antibodies were goat polyclonal anti-RRM2 antibody (Santa Cruz Biotechnology), and mouse polyclonal anti-actin antibody (BD Biosciences). The secondary antibodies were horseradish-peroxidase-conjugated donkey anti-goat antibody, and rabbit antimouse antibody (Santa Cruz Biotechnology). Development was done using SuperSignal West Dura Extended Duration Substrate (Thermo-Fisher). Blot images were captured using a Molecular Imager VersaDoc 3000 system (BioRad). Band quantification was performed using Image-Quant TL software (GE/ Amersham Biosciences).

Tissues and immunohistochemical assay. Formalin-fixed, paraffin-embedded human tissue samples from patient-matched pre- and post-treatment cases were obtained under UCLA Institutional Review Board (IRB) approval. IHC assays were performed using a Dako Autostainer Plus (Dako) with fresh sections of pre- and post-treatment cases stained at the same time. Tissue sections $4-\mu \mathrm{m}$ thick were deparaffinized in xylene and rehydrated in graded alcohols. The sections were then placed in a pressure cooker $\left(17.5\right.$ PSI, $122^{\circ} \mathrm{C}$; Biocare Decloaking Chamber, Biocare Medical) in $0.01 \mathrm{M}$ sodium citrate buffer, $\mathrm{pH} 6.0$, or $0.1 \mathrm{M}$ Tris- $\mathrm{HCl}$ buffer, $\mathrm{pH} 9.0$, for $10 \mathrm{~min}$ for heat-antigen retrieval of RRM 2 and TFR antigens, respectively. Endogenous peroxidase was quenched with 3\% hydrogen peroxide at room temperature. Primary goat anti-human R2 polyclonal antibody (sc-10846; Santa Cruz Biotechnology), was applied for $30 \mathrm{~min}$ at room temperature at a final concentration of $1.0 \mu \mathrm{g} \mathrm{ml}^{-1}$ (1:200). Mouse anti-human TFR monoclonal IgG1 antibody (clone H68.4, 13-6800; Invitrogen) was applied for $30 \mathrm{~min}$ at room temperature at a final concentration of $0.5 \mu \mathrm{g} \mathrm{ml}^{-1}(1: 1,000)$. Antigen detection was accomplished using the Vectastain ABC Elite Goat HRP kit (Vector Labs) or the Dako Envision goat anti-mouse IgG secondary antibody with attached HRP-labelled dextran polymer (K4001; Dako), for RRM2 and TFR, respectively. All tissues were either amelanotic or only lightly melanized, therefore bleaching was not performed and Nova Red (Vector Labs) was used as the chromagen to easily discern staining from any endogenous pigment. The sections were then counterstained with Meyer's haematoxylin, followed by dehydration in graded alcohols, xylene and the addition of a coverslip. Human tonsil and colon cancer served as positive assay controls. Negative controls consisted of duplicate tissue sections stained with either non-immune pooled goat IgG (I-5000, Vector Labs) or monoclonal mouse IgG1 (02-6100; Invitrogen), applied at identical final concentrations as used for RRM2 and TFR primary antibodies, respectively. For each sample, ten random tumour regions were scored for maximal expression and mean expression. 